\title{
Tracheal Lymphoma
}

National Cancer Institute

\section{Source}

National Cancer Institute. Tracheal Lymphoma. NCI Thesaurus. Code C6248.

A rare lymphoma that arises from the trachea. Signs and symptoms include dyspnea, cough, wheezing, and stridor. 